\title{
A theorem on homeomorphism groups and products of spaces
}

\author{
A. R. Vobach
}

Let $H(C)$ be the group of homeomorphisms of the Cantor set, $C$, onto itself. Let $p: C \rightarrow M$ be a map of $C$ onto a compact metric space $M$, and let $G(p, M)$ be $\{h \in H(C) \mid \forall x \in C, p(x)=p h(x)\} . G(p, M)$ is a group. The map $p: C \rightarrow M$ is standard, if for each $(x, y) \in C \times C$ such that $p(x)=p(y)$, there is a sequence $\left\{x_{n}\right\}_{n=1}^{\infty} \subset C$ and a sequence $\left\{h_{n}\right\}_{n=1}^{\infty} \subset G(p, M)$ such that $x_{n} \rightarrow x$ and $h_{n}\left(x_{n}\right) \rightarrow y$. Standard maps and their associated groups characterize compact metric spaces in the sense that: Two such spaces, $M$ and $N$, are homeomorphic if and only if, given $p$ standard from $C$ onto $M$, there is a standard $q$ from $C$ onto $N$ for which $G(p, M)=h^{-1} G(q, N) h$, for some $h \in H(C)$

The present paper exhibits a structure theorem connecting these characterizing subgroups of $H(C)$ and products of spaces: Let $M_{1}$ and $M_{2}$ be compact metric spaces. Then there are standard maps $p: C \rightarrow M_{1} \times M_{2}$ and $p_{i}: C \rightarrow M_{i}, i=1,2$, such that $G\left(p, M_{1} \times M_{2}\right)=G\left(p_{1}, M_{1}\right) \cap G\left(p_{2}, M_{2}\right)$.

The following definition and results were given in [1]:

Let $H(C)$ be the group of homeomorphisms of the Cantor set, $C$, onto itself. Let $p: C \rightarrow M$ be a map (continuous) of $C$ onto a compact metric

Received 2l March 1969. Received by J. Austral. Math. Soc. 10 May 1966. Communicated by G.B. Preston. This research was partly supported by NSF Grant No. Gp 3915. 
space $M$, and let $G(p, M)=\{h \in H(C) \mid \forall x \in C, p(x)=p h(x)\}$. $G(p, M)$ is a group.

A map, $p$, of $C$ onto $M$ is a standard map, if for each pair of points, $x$ and $y$, such that $p(x)=p(y)$, there is a sequence $\left\{x_{n}\right\}_{n=1}^{\infty} \subset C$ and a sequence $\left\{h_{n}\right\}_{n=1}^{\infty} \subset G(p, M)$ such that $x_{n} \rightarrow x$ and $h_{n}\left(x_{n}\right) \rightarrow y$. Standard maps are very naturally obtained and their groups characterize compact metric spaces in the following sense: Two compact metric spaces, $M$ and $N$, are homeomorphic if and only if, given $p$ standard from $C$ onto $M$, there is a standard $q$ from $C$ onto $N$ for which $G(p, M)=h^{-1} G(q, N) h$, for some $h \in H(C)$.

The following theorem is intended to exhibit a natural connection between these characterizing subgroups of $H(C)$ and products of compact metric spaces.

THEOREM. Let $M_{1}$ and $M_{2}$ be compact metric spaces. Then there are standard maps $p: C \rightarrow M_{1} \times M_{2}$ and $p_{i}: C \rightarrow M_{i}, i=1,2$, such that $G\left(p, M_{1} \times M_{2}\right)=G\left(p_{1}, M_{1}\right) \cap G\left(p_{2}, M_{2}\right)$.

While the following hardy deserves to be called a lemma, it is inserted before the proof of the theorem to simplify subsequent constructions.

LEMMA. If $p: C_{2} \rightarrow M$, a compact metric space, is a standard map, and $h: C_{1} \rightarrow C_{2}$ is a homeomorphism, then $p h: C_{1}+M$ is a standard map.

Proof of Lemma. Suppose, for $x, y \in C_{1}, p h(x)=p h(y)$. Let $\boldsymbol{z}=h(x)$ and $w=h(y)$, so that $p(z)=p(w)$. Then, from the standardness of $p$, there exist sequences $\left\{x_{n}\right\}_{n=1}^{\infty} \subset C_{2}$ and $\left\{f_{n}\right\}_{n=1}^{\infty} \subset G(p, M)$ such that $x_{n}+z$ and $f_{n}\left(x_{n}\right) \rightarrow w$. If $s_{n}=h^{-1}\left(x_{n}\right)$, then $h\left(s_{n}\right) \rightarrow h(x)=z$ and $s_{n} \rightarrow x$. Defining $\left\{g_{n}=h^{-1} f_{n} h\right\}_{n=1}^{\infty}$, observe that, for $v \in C_{1}, p h g_{n}(v)=p h\left(h^{-1} f_{n} h\right)(v)=p f_{n}(h(v))=p h(v)$, so that $\left\{g_{n}\right\}_{n=1}^{\infty} \subset G(p h, M)$. Also $g_{n}\left(s_{n}\right)=h^{-1} f_{n}\left(x_{n}\right)+h^{-1}(w)=y$.

Proof of Theorem. Let $C_{1}$ and $C_{2}$ be Cantor sets; let $h: C \rightarrow C_{1} \times C_{2}$ be a homeomorphism, and let $q_{i}: C_{i}+M_{i}, i=1,2$, 
be standard maps. With $\Pi_{i}: c_{1} \times c_{2} \rightarrow c_{i}$ defined by $\Pi_{i}\left(y_{1}, y_{2}\right)=y_{i}$, $i=1,2$, let $p: C \rightarrow C_{1} \times C_{2} \rightarrow M_{1} \times M_{2}$ be defined by $p(x)=\left(q_{1} \Pi_{1} h(x), q_{2} \Pi_{2} h(x)\right)$. Clearly, $p$ is continuous onto $M_{1} \times M_{2}$. Next we see that each $p_{i}=q_{i} \Pi_{i} h$ is a standard map: We first show that $q_{i} \Pi_{i}: C_{1} \times C_{2} \rightarrow M_{i}$ is standard and then remark that, by the Lemma, $q_{i} \Pi_{i} h$ is still a standard map. Let $q_{i} \Pi_{i}\left(x_{1}, x_{2}\right)=q_{i} \Pi_{i}\left(y_{1}, y_{2}\right)$; then standardness of $q_{i}$ says there is a sequence $\left\{z_{n}=\Pi_{i}\left(w_{1, n}, w_{2, n}\right)\right\}_{n=1}^{\infty} \subset c_{i}$ and a sequence $\left\{h_{n}^{\prime}\right\}_{n=1}^{\infty} \subset G\left(q_{i}, M_{i}\right)$ such that $z_{n}=w_{i, n} \rightarrow x_{i}$ and $h_{n}^{\prime}\left(w_{i, n}\right) \rightarrow y_{i}$.

From now on, it will be notationally convenient to work with a particular choice of $i$, say $i=1$. Let $w_{2, n}=x_{2}$ for the ordered pairs above we are only interested in the projection onto the first coordinate - and let $h_{0}: C_{2} \rightarrow C_{2}$ be a homeomorphism for which $h_{0}\left(x_{2}\right)=y_{2}$. Now let $h_{n}: C_{1} \times C_{2} \rightarrow C_{1} \times C_{2}$ be defined by $h_{n}\left(v_{1}, v_{2}\right)=\left(h_{n}^{\prime}\left(v_{1}\right), h_{0}\left(v_{2}\right)\right)$, so that $\left(z_{n}, x_{2}\right) \rightarrow\left(x_{1}, x_{2}\right)$ and $h_{n}\left(z_{n}, x_{2}\right) \rightarrow\left(y_{1}, y_{2}\right)$. We may claim $h_{n}, n=1, \ldots$, is in $G\left(q_{1} \Pi_{1}, M_{1}\right)$ because

$$
\begin{aligned}
q_{1} \Pi_{1}\left(v_{1}, v_{2}\right) & =q_{1}\left(v_{1}\right) \\
& =q_{1} h_{n}^{\prime}\left(v_{1}\right) \\
& =q_{1} \Pi_{1}\left(h_{n}^{\prime}\left(v_{1}\right), h_{0}\left(v_{2}\right)\right) \\
& =q_{1} \Pi_{1} h_{n}\left(v_{1}, v_{2}\right) .
\end{aligned}
$$

The proof for $q_{2} \Pi_{2}$, with $i=2$, is obviously similar. As noted, $q_{i} \Pi_{i} h, i=1,2$, is also standard.

Next, we must show that $p: C \rightarrow C_{1} \times C_{2} \rightarrow M_{1} \times M_{2}$ is standard: It suffices to show standardness of $q: h(C)=C_{1} \times C_{2} \rightarrow M_{1} \times M_{2}$ defined by $q\left(v_{1}, v_{2}\right)=\left(q_{1}\left(v_{1}\right), q_{2}\left(v_{2}\right)\right)$. Suppose $q\left(x_{1}, x_{2}\right)=q\left(y_{1}, y_{2}\right) ;$ this means $q_{1}\left(x_{1}\right)=q_{1}\left(y_{1}\right)$ and $q_{2}\left(x_{2}\right)=q_{2}\left(y_{2}\right)$. Standardness of $q_{1}$ implies there exist sequences $\left\{z_{n}\right\}_{n=1}^{\infty} \subset C_{1}$ and $\left\{f_{n}\right\}_{n=1}^{\infty} \subset G\left(q_{1}, M_{1}\right)$ such that $z_{n}+x_{1}$ and $f_{n}\left(z_{n}\right) \rightarrow y_{1}$. Likewise, there exist sequences $\left\{w_{n}\right\}_{n=1}^{\infty} \subset c_{2}$ 
and $\left\{g_{n}\right\}_{n=1}^{\infty} \subset G\left(q_{2}, M_{2}\right)$ such that $w_{n} \rightarrow x_{2}$ and $g_{n}\left(w_{n}\right) \rightarrow y_{2}$.

Consider the sequence $\left\{\left(z_{n}, w_{n}^{\prime \prime}\right\}_{n=1}^{\infty} \subset C_{1} \times C_{2} ;\right.$ since $z_{n} \rightarrow x_{1}$ and $w_{n} \rightarrow x_{2}, \quad\left(z_{n}, w_{n}\right) \rightarrow\left(x_{1}, x_{2}\right)$. Let $h_{n}: C_{1} \times C_{2}$ be defined by $h_{n}\left(v_{1}, v_{2}\right)=\left(f_{n}\left(v_{1}\right), g_{n}\left(v_{2}\right)\right)$. Then $h_{n}\left(z_{n}, w_{n}\right) \rightarrow\left(y_{1}, y_{2}\right)$. We claim $h_{n}, n=1,2, \ldots$, is in $G\left(q, M_{1} \times M_{2}\right)$ because

$$
\begin{aligned}
q\left(v_{1}, v_{2}\right) & =\left(q_{1} \Pi_{1}\left(v_{1}, v_{2}\right), q_{2} \Pi_{2}\left(v_{1}, v_{2}\right)\right) \\
& =\left(q_{1} f_{n} \Pi_{1}\left(v_{1}, v_{2}\right), q_{2} g_{n} \Pi_{2}\left(v_{1}, v_{2}\right)\right) \\
& =\left(q_{1} f_{n}\left(v_{1}\right), q_{2} g_{n}\left(v_{2}\right)\right) \\
& =\left(q \Pi_{1}\left(f_{n}\left(v_{1}\right), g_{n}\left(v_{2}\right)\right), q_{2} \Pi_{2}\left(f_{n}\left(v_{1}\right), g_{n}\left(v_{2}\right)\right)\right) \\
& =\left(q_{1} \Pi_{1} h_{n}\left(v_{1}, v_{2}\right), q_{2} \Pi_{2} h_{n}\left(v_{1}, v_{2}\right)\right) \\
& =q h_{n}\left(v_{1}, v_{2}\right),
\end{aligned}
$$

each $\left(v_{1}, v_{2}\right) \in C_{1} \times C_{2}$.

Since $h$ is a homeomorphism, $p=q h$ is also standard.

Finally, $G\left(p, M_{1} \times M_{2}\right)=G\left(p_{1}, M_{1}\right) \cap G\left(p_{2}, M_{2}\right)$ : First, for $f \in G\left(p, M_{1} \times M_{2}\right)$ and each $x \in C$,

$$
\begin{aligned}
p(x) & =\left(q_{1} \Pi_{1} h(x), q_{2} \Pi_{2} h(x)\right) \\
& =p f(x) \\
& =\left(q_{1} \Pi_{1} h f(x), q_{2} \Pi_{2} h f(x)\right),
\end{aligned}
$$

which says $q_{i} \Pi_{i} h(x)=q_{i} \Pi_{i} h f(x), i=1,2$, and $f \in G\left(p_{1}, M_{1}\right) \cap G\left(p_{2}, M_{2}\right)$. Second, for $f \in G\left(p_{1}, M_{1}\right) \cap G\left(p_{2}, M_{2}\right)$ and each $x \in C$,

$$
\begin{aligned}
p(x) & =\left(q_{1} \Pi_{1} h(x), q_{2} \Pi_{2} h(x)\right) \\
& =\left(q_{1} \Pi_{1} h f(x), q_{2} \Pi_{2} h f(x)\right) \\
& =p f(x)
\end{aligned}
$$

and $f \in G\left(p, M_{1} \times M_{2}\right)$. 


\section{Reference}

[1] Arnold R. Vobach, "On subgroups of the homeomorphism group of the Cantor set", Fund. Math. 60 (1967), 47-52.

University of Georgia,

Athens, Georgia

and

University of Houston,

Houston, Texas. 